\title{
Electronic properties and quasi-zero-energy states of graphene quantum dots
}

\author{
H. V. Grushevskaya* and G. G. Krylov ${ }^{\dagger}$ \\ Physics Department, Belarusian State University, 4 Nezalezhnasti Ave., 220030 Minsk, Belarus \\ S. P. Kruchinin \\ Bogolyubov Institute for Theoretical Physics, Metrologichna str. 14-b, 03143 Kiev, Ukraine \\ B. Vlahovic \\ North Carolina Central University, Durham, NC, USA \\ Stefano Bellucci \\ INFN-Laboratori Nazionali di Frascati, 54 Via E. Fermi, 00044 Frascati, Italy
}

\begin{abstract}
In this work, a research has been carried out into the electronic properties of nanostructured graphene. We focus our attention on trapped states of the proposed systems such as spherical and toroidal graphene quantum dots. Using a continuum model, by solving the Dirac-Weyl equation, and applying periodic boundary conditions of two types, i.e. either with zigzag-edges only, or with both armchair- and zigzag-edges, we obtain analytical results for energy levels yielding self-similar energy bands located subsequently one after another on the energy scale. Only for toroidal quantum dots (owing to the lack of curvature) the distribution of electron density is similar to Bohr atomic orbitals. However, although the quasi-zero-energy band exists for both spherical and toroidal quantum dots, no electron density is present on this band for the toroidal quantum dot. This causes the formation of a pseudogap between the hole and electron bands, because of the absence of the electron density at the quantum dot center, like in the case of an ordinary atom. Conversely, the confinement of the charge-carrier density is observed for both geometries of graphene quantum dots.
\end{abstract}

\section{INTRODUCTION}

Nanodimensional monolayer graphene patches are promising as a basis for the development of quantum devices. Graphene physics is the physics of massless charge carriers. The nanometre-sized quasi-circular graphene monolayer samples, called graphene quantum dots (GQDs), can be chemically synthesized. Chemically synthesized nanoscale GQDs have the form of a quasicircle with a radius in the interval from $3 \mathrm{~nm}$ to $10 \mathrm{~nm} \mathrm{[1].} \mathrm{By} \mathrm{scattering} \mathrm{on} \mathrm{the} \mathrm{physical} \mathrm{edges} \mathrm{of}$ contacting graphene patches, charge carriers drastically impair their mobility. Moreover, the fact that the Schrödinger equation with a confining parabolic potential satisfactorily describes at least the low-lying states of the synthesized dots means that the distortion of electronhole physics is possible up to the loss of bipolarity of the material (see e.g., [2, 3] and references therein).

The problem of contacts in graphene electronics can be solved by designing the so-called electrically confined graphene quantum dots without physical termination. The electrically confined GQD is a part of the graphene monolayer, separated by a potential barrier from the rest of the monolayer. The electrostatic geometric confinement in a graphene monolayer allows to finely tune the charge localization and scattering in graphenebased devices. To form an electrically confined GQD, the

\footnotetext{
*Electronic address: grushevskaja@bsu.by

${ }^{\dagger}$ Electronic address: krylov@bsu.by
}

graphene monolayer deposited on the support is $n(p)$ doped. The local action of an electric field (screening) directed opposite to the electric field of $n(p)$ doping changes locally the type of doping from $n(p)$ to $p(n)$. The resulting $n-p$ or $p-n$ junction is an electrically confined GQD. The $n(p)$ doping of a bulk monolayer can be achieved by applying an electric voltage $V_{B G}$ to the support.

The design of nanoelectronic devices based on graphene $n-p(p-n)$ junctions requires the knowledge of model-independent realistic confining electrostatic potentials. Model confining potentials for GQDs have been currently offered. However, their shape is determined by the specificity of fabrication method. There are three techniques. First, an electric field of the graphene-doping electrode (backgate) contacting with the support can be locally screened by forming an additional thin (needle-like) metal structure on the surface of the tip of the scanning tunneling microscope (STM) [4, 5]. Such quantum dots are called tip-induced ones. Second, under the local action of an electric voltage pulse $V_{T G}$ on the STM tip, the nanometersized graphene region is doped with charge carriers of the opposite sign with respect to bulk graphene [6]. A mechanism for creating a local $\mathrm{p}(\mathrm{n})$-doped region by the second method is the ionization of defects in the hexagonal boron-nitride (h-BN) layer of support by the electric-field pulse, the released (migrated) charges screen the electric field acting from the side of the support, that is equivalent to local $\mathrm{p}(\mathrm{n})$-doping. In this case, the support plays a role of a backgate, the local $\mathrm{p}(\mathrm{n})$-doping is equivalent to a fabrication of local embedded gate. Third, the shielding 
embedded gate can be effectively formed as a part of metal support, which remains defect-free during epitaxial growth of graphene on its surface [7]. The advantage of the field doping used in the first two methods is the absence of a Fermi level shift under the action of the chemical potential.

The confining potential of the tip-induced GQD is subjected to strong influence of electric fields due to peculiarities of the field spatial configuration, schematically presented in fig. 1a. Since the screened region is bound to the STM double-tip and, correspondingly, the tip-induced GQD functionally is bound to the tip, while a structure of such junctions was not observed in the images. The atomic-like nature of these graphene $p-n$ junctions is revealed in the experimental dependence of a derivative of the tunneling current $d I / d V$ on voltages $V_{T G}$ and $V_{B G}$. The dependence is similar to local density of states (LDOS) for a tight-binding Hamiltonian of graphene charge carriers moving in a potential of the Thomas-Fermi atom model [5]. According to this estimate, the quantum dot is an artificial atom with a huge number of electrons, as the effective electric charge of Coulomb potential or the Coulomb coupling $\beta=\left(Z / \epsilon_{G}\right) \alpha c / v_{F}$, which confines charge carriers in the tip-induced junction, takes on values typical for a supercritical regime of ultra-heavy atoms. Here $Z$ is a local unscreened charge, $c$ is the speed of light, $v_{F}$ is the Fermi velocity and $\epsilon_{G}$ is the effective dielectric constant of bulk graphene, $\epsilon_{G} \approx 5$ [8]. The atomic-like distribution of the electron (hole) density in such quantum dots is fitted by a continuous Thomas-Fermi-like approximation for a potential $U(r)$ confining electrons (holes): $U(r)=\operatorname{sgn}(n) \hbar v_{F} \sqrt{\pi|n(r)|}$. Here $n(r)$ is a charge density (for holes in p-n junctions or for electrons in $n-p$ junctions). The confining model potential based on the Thomas-Fermi model is two-step potential [5], and ab-initio full tight-binding calculations with a Poisson's equation predict a stair-case form for the confining potentials (see fig. 1b) [4, 9, 10].

Since the backgate and the created embedded gate exist independently of the STM-tip, the atom-like structure of GQDs fabricated by the 2nd and 3rd methods can be imaged by a STM probing of their LDOS using voltages that practically do not change the initial configuration of the gate electric fields shown schematically in fig. 1c. The imaging allows to construct the confining electrostatic potential directly from the experimental values of the touchings $E_{D}^{(i)}, i=1,2, \ldots$. between Dirac graphene valence and conduction bands. For example, a radial step potential is constructed on the basis of two values of $E_{D}$ inside and outside the smallradius quantum dot (see fig. 1d). A parabolic potential as an infinite set of single-step radial potentials is used to describe large GQDs (see fig. 1e). When constructing a model confining potential for the third fabricating method, it is also necessary to anticipate an offset of the chemical potential $\mu$ for GQD after doping of graphene with metal atoms.
A feature of the scattering Dirac fermions with mass $m$ is the existence of delocalized modes among scattered ones under the condition $V>2 m$ imposed on the potential barrier $V$ [11]. The delocalized modes are called transmission resonances, and the tunneling process is called Klein's one (see also [12] and references therein). The fermions pass freely through the potential barrier at normal incidence on the boundary of the barrier $V$ or phases that are multiples of $\pi \pm 2 \pi n ; n=0,1, \ldots$ [13]. For the massless graphene fermions, the transmission resonances will always be present among the scattered modes. Assuming that the effects of Klein tunneling will always distort the electrostatic confinement of massless charge carriers, the variety of solutions to the problem of pseudo-Dirac fermions in electrostatically confined graphene $\mathrm{p}-\mathrm{n}(\mathrm{n}-\mathrm{p})$ junctions can be narrowed down to the subvariety of levels localized near the Fermi level (quasi-zero-energy levels or whispering gallery modes). Due to the orbital motion of charge carriers a production of electron-hole pairs does not happen. Unfortunately, for the massless fermions a condition selecting eigenstates which are localized at the edge of the radial step barrier and behave like to Dirac $\delta$ function, gives a single level or has unphysical solutions at all, depending on the GQD parameters [14, 15]. In [7], the set of states with one real eigenvalue is speculatively expanded by adding solutions with complex eigenvalues, manipulating (juggling) with them to achieve an apparent similarity between calculated LDOS and $d I / d V$. The nonrelativistic tight-binding Hamiltonians, which fundamentally do not possess Klein scattered modes, narrow the possible types of edge spatial configurations at which there is a zero-energy level in the quasi-circular GQD [16]. It turns out that the spectra of zigzag-edged quasi-circular GQDs (zigzag edges at the boundary prevail) and zigzag-edged hexagonal graphene quantum dots host only one zero-energy level, and the tight-binding graphene quantum dot model of arbitrary shape does not predict the modes localized at the boundary of the cavity [17-20]. A simplified continuous pseudo-Dirac Hamiltonian with a radial step potential hosts a zero eigenvalue at the zigzag-boundary condition. But among eigenvalues of the Hamiltonian there are no zero- or quasizero-energies at the so-called infinite-mass boundary condition meaning that the region outside the dot is forbidden or the type of all edges is an armchair one and, correspondingly, the boundary is non-metallic $[18,21,22]$. Another mechanism for the emergence of the zero-energy level is associated with exciton instability in graphene [23]. It can be assumed that electron-hole pairs become Keldysh-type exciton states, energetically favorable in a low-dimensional system with the dielectric constant $\epsilon$ (in the case, one dimension). The screened electron-hole interactions added to the continuous pseudo-Dirac fermion model with a radial step potential at the infinite-mass boundary condition result in a zero energy level [24]. Unfortunately, even when using the low dielectric constant $\epsilon_{G}=2.5$ (while 
(b)

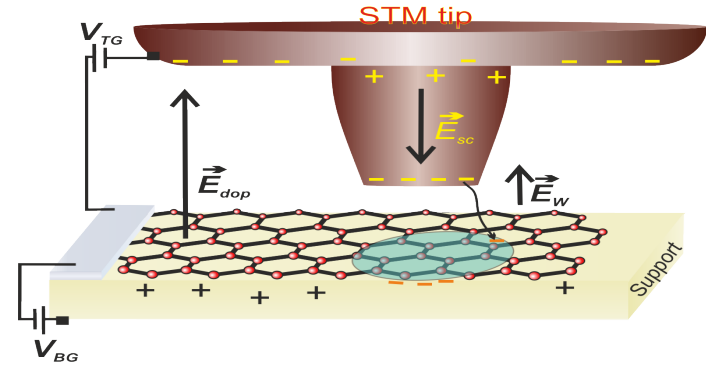

(c)

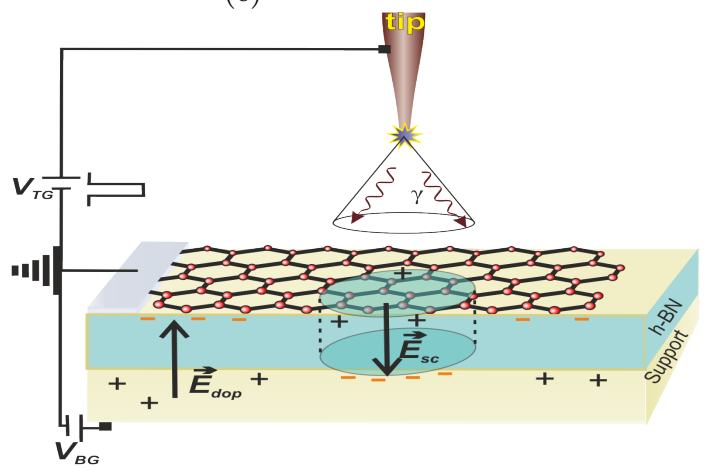

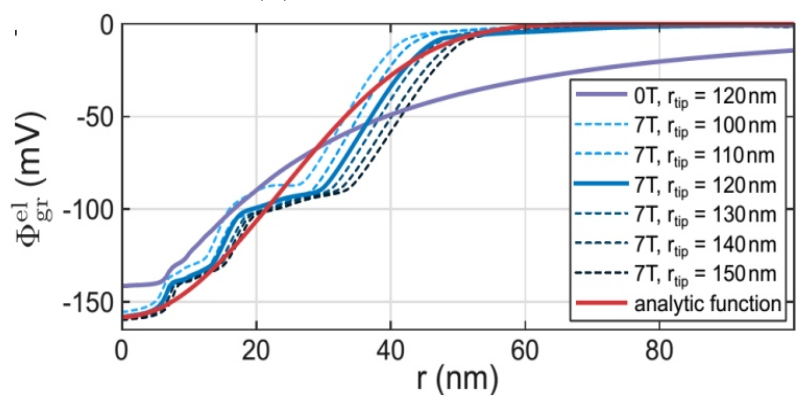

(d) (e)

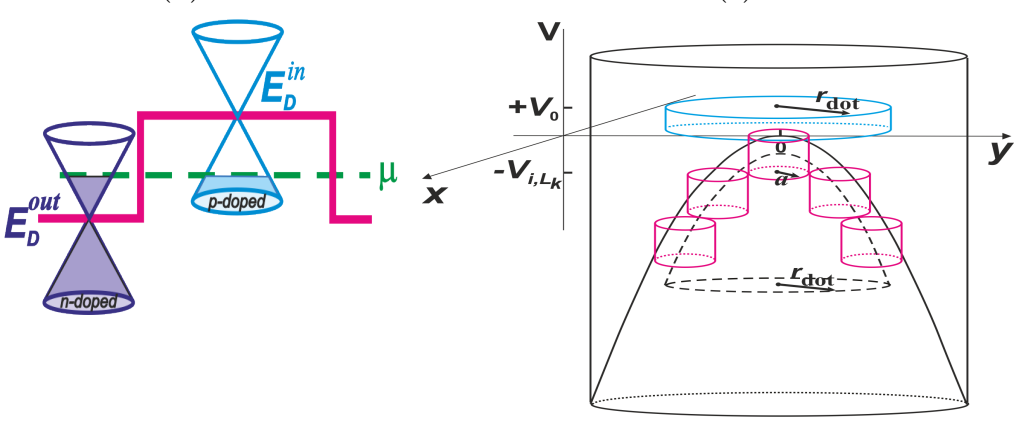

FIG. 1: A sketch of electrical fields creating circular graphene n-p and p-n junctions and the model potentials confining charge carriers in the junctions. (a) The n-p junction is induced by a STM double tip, which consists of a large-radius tip and a small-radius tip attached to the latter at the lowest point. Emerging at application of voltages $V_{T G}$ and $V_{B G}$ to the STM tip (top gate) and support (back gate) an electric field $\vec{E}_{d o p}$ dopes the bulk graphene with a positive charge. By polarizing the metallic thin tip one creates an electric field $\vec{E}_{s c}$, which screens the graphene region located under the tip from the action of $\vec{E}_{d o p}$, that locally results in changing p-doping to n-doping. The level of n- doping also depends on the electric field $\vec{E}_{W}=\Delta \vec{W} / e$ produced by the difference between work functions $W_{g}$ and $W_{t}$ of graphene and metal probe tip; (b) Cos-shape potential reconstructed on experimental data [4]; (c) The p-n junction is induced by a pulse of STM-tip voltage. The electromagnetic quanta $\gamma$ polarize the h-BN region directly underneath the tip. The electric field $\vec{E}_{s c}$ in this h-BN region functioning as a negatively charged local embedded gate positively dopes the graphene region underneath the tip. $\vec{E}_{s c}$ screens the p-doped graphene region from an action of the electric field $\vec{E}_{\text {dop }}$ which dopes bulk graphene with a negative charge; (d) Schematic of confining-potential measurements over two Dirac band touchings $E_{D}^{\text {in }}$ and $E_{D}^{\text {out }}$ inside and outside electrostatically confined graphene p-n junction respectively. The Dirac-touching offset produces a radial step potential $V(r)$. The chemical potential $\mu$ is marked by a dashed line; (e) Model scattering potentials for the graphene quantum dot of radius $r_{\text {dot: a long- }}$ wave approximation $V_{i, R_{k}} \propto-\epsilon_{R_{k}}\left(q_{i}\right) \Theta\left(\lambda_{i}-R\right) \Theta(a-r)$ of pseudopotential (II.20) (red curves), $R_{k}=|\vec{R}-\vec{r}|$; a parabolic potential $\left(-\kappa r^{2}\right)$ with a large repulsive potential outside the graphene quantum dot (black curves); a cylindrical barrier potential $V=+V_{0} \Theta\left(r_{\text {dot }}-R\right) \geq 0, V_{0}>0$ (blue curves). The,+- signs indicate the charge polarities in the tip and in graphene with support.

experiments give $\epsilon_{G}=5$ ), the exciton binding energy is very small at least 2 times less (about $50 \mathrm{meV}$ ), than the probing voltage (electrostatic potential) $0.1-$ $1 \mathrm{eV}$. Therefore, the exciton polarization cannot be the mechanism responsible for the existence of zero-energy states.

Thus, the problem of existing zero-energy level for a graphene junctions with arbitrary edged configurations is still unsolved. Moreover, the stability of the experimentally observed quasi-zero-energy levels can be not only and not that much related to the peculiarities of the Klein tunneling process. There are experimental indications that long-lived scattered resonances can be at deep enough levels of confining stair-case potentials
[5]. Also large p-n (n-p) junctions fabricated by the 2nd technique are characterized by a huge number of quasi-stationary levels far from the GQD edge. This could be an indication that the GQD can exist for quite a long time in states other than quasi-zero-energy modes. The confinement of massless fermions in the multi-step potential can be considered simplistically as a confinement of pseudoDirac fermion in a parabolic potential. Unfortunately, since the quasi-zero-energy levels at the edge of such a GQD are spurious ones, the assignment of the levels in the parabolic confining potential to the long-lived Klein resonances cannot be considered as correctly justified [8].

Generally speaking, since GQDs are many-body 
artificial atoms, their models must have a lot of quasizero energy modes. The phase condition for the existence of transmission resonances has nothing common with their spatial configuration and can expand the search for the solutions to the region of Klein transmission modes delocalized in a limited GQD region. In addition, the experimental LDOS of electrostatically confined junctions is polarized [6]. Until now, no models of such exotic states have been proposed. The amazing fact that the modern GQD models cannot have quasi-zero-energy levels, except for the actual zero-energy level, should be interpreted as a huge problem, without a solution to which there can be no talk of physics of graphene quantum dots.

So, the search for selection criteria of GQD with quasi-zero-energy levels and transmission resonances, delocalized in a limited spatial area, is a difficult and unsolved problem. The lack of models, in which a quasizero-energy band emerges, prevents the development of methods to tune up the graphene junction in a way that its modes do not turn out to be short-living resonances, and Klein transmission resonances are delocalized in a limited area of space. Therefore, a realization of devices based on the tailored charge confinement in a graphene monolayer is still challenging.

In this paper we simulate electrostatically confined graphene quantum dots, using a quasirelativistic tightbinding Hamiltonian of massless fermions in monolayer graphene. We present a discrete model of graphene quantum dot and its continuous limit to describe realistic electrostatically confined graphene quantum dots. To do this, we use a high-energy $\vec{k} \cdot \vec{p}$ masslessfermions Hamiltonian. A band structure obtained from the Hamiltonian holds the Dirac touching and six Weyl nodes-antinodes pairs as minibands near valley $K\left(K^{\prime}\right)$ of graphene Brillouin zone. Then we look for a pseudopotential barrier given by a set of well potentials for distinct carbon atoms of the graphene quantum dot. We discover two topologically different scenarios of the confinement in a quantum dot.

\section{MODEL OF ELECTRICALLY-CONFINED GRAPHENE QUANTUM DOT AND THEORY}

A graphene monolayer quantum dot consisting of carbon atoms is shown schematically in fig. 2a. Let a model quantum dot be considered as a "large atom". Its core $i$-th electrons, by definition, are $\mathrm{p}_{z}$-electrons of $j$-th $\mathrm{C}$ atoms, $j \neq i$. The $k$-th $\mathrm{p}_{z}$-electron of $k$-th $\mathrm{C}$ atom plays the role of an external valence electron. Let the $k$-th $\mathrm{C}$ atom be placed at the lattice site with a radius-vector $\vec{R}_{k}$. The radius vector $\vec{r}$ will be calculated on respect to the nearest lattice site and is a radius vector of the electron in the atom. The radius vector $\vec{X}_{k}$ of the valence electron of $k$-th atom is given by the expression

$$
\vec{X}_{k}=\vec{R}_{k}+\vec{r}
$$

A model graphene quantum dot has been constructed in the following way. The graphene primitive cell has basic vectors $\vec{b}_{1}=a(3 / 2, \sqrt{3} / 2), \quad \vec{b}_{2}=a(3 / 2,-\sqrt{3} / 2)$ and two atoms (A and $\mathrm{B}$ ) in the cell. Here $a$ is the length of $\mathrm{sp}^{2}$-hybridized $\mathrm{C}-\mathrm{C}$ bond. We construct a rhombic region consisting of $\left(2 n_{1}+1\right) *\left(2 n_{2}+1\right)$ primitive cells of graphene for $n_{1}=25, n_{2}=25$, that is shown in fig. $2 \mathrm{~b}$.

\section{A. "Folding zone" approximation}

The energy levels of a graphene quantum dot can be approximately found by the "folding-zone" method in the same way as for single walled carbon nanotubes (CNTs). Electronic states of CNTs are restricted to wave vectors that fulfill the quantization condition [25]:

$$
\vec{k}_{\perp} \cdot \vec{C}=2 \pi m, \quad m=-N / 2+1, \ldots, N / 2
$$

where $\vec{k}_{\perp}$ is a wavevector in the direction perpendicular to the CNT axis; $\vec{C}$ is a chiral vector of CNT, $N$ is the number of graphene hexagonal unit cells within a CNT unit cell, $m$ is an index of one-dimensional (1D) band. The condition (II.2) is the so called "Born-von-Karman" condition.

Contrary to the CNT case, the wavevector $\vec{k}=$ $\left(k_{x}, k_{y}\right)$ of a charge carrier in the dot is quantized in two directions. Moreover, the quantum dots can be constructed with topologies: sphere $S^{2}$ and torus $S^{1} \times S^{1}$ in the following way. The dot topologically equivalent to sphere is formed by rhombuses inscribed into rectangular supercells. Such a geometrical structure can be obtained by a shift on half-period of rectangular lattice. The sphere topology is obtained by identifying all vertices of the rhombus and gluing adjusting its sides. In the case of rectangular unit cell a quantum dot can be produced, for example, by 4 or 8 supercells. The quantum dots of this type are presented in figs. 2f,g. All edges of 8-supercell dot are in armchair configurations. The edges of 4-supercell dot are both in armchair and zigzag configurations. Since the symmetry group for the superlattice with rectangular unit cell is non-symmorphic one, there are no punctured points.

Since the basis vectors of the rectangular unit are $\vec{C}_{1}=$ $\left(2 n_{1}+1\right) \vec{b}_{1}+\left(2 n_{2}+1\right) \vec{b}_{2}$ and $\vec{C}_{2}=\left(2 n_{1}+1\right) \vec{b}_{1}-\left(2 n_{2}+\right.$ 1) $\vec{b}_{2}$, quantization conditions for the $S^{2}$ type graphene quantum dot read

$$
\begin{array}{r}
\left(\left(2 n_{1}+1\right) \vec{b}_{1}+\left(2 n_{2}+1\right) \vec{b}_{2}\right) \cdot \vec{k}=2 \pi m_{1}, \\
m_{1}=-N_{d o t} / 2+1, \ldots, N_{d o t} / 2 ; \\
\left(\left(2 n_{1}+1\right) \vec{b}_{1}-\left(2 n_{2}+1\right) \vec{b}_{2}\right) \cdot \vec{k}=2 \pi m_{2}, \\
m_{2}=-N_{d o t} / 2+1, \ldots, N_{d o t} / 2 .
\end{array}
$$


(a)

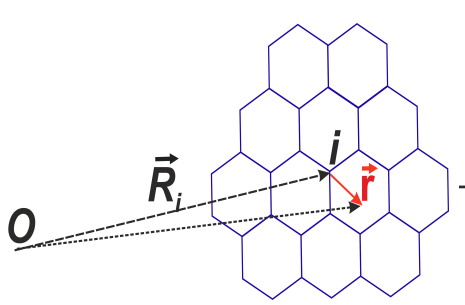

(d)

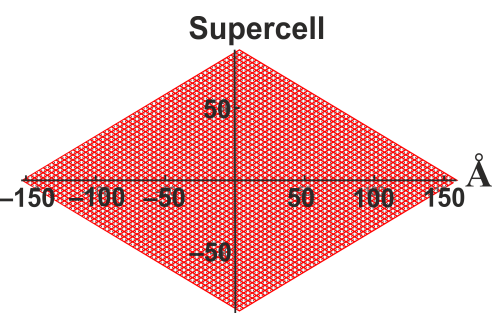

(e) (b)

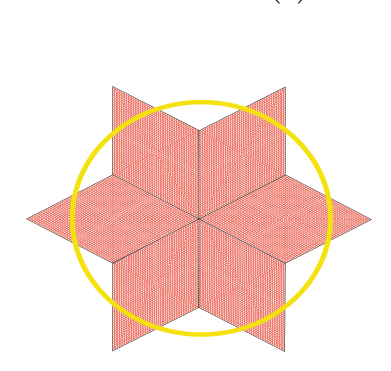

(f)

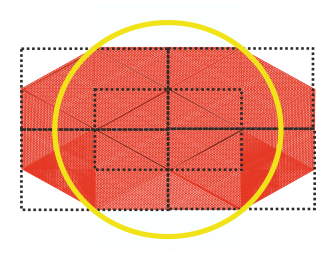

(c)

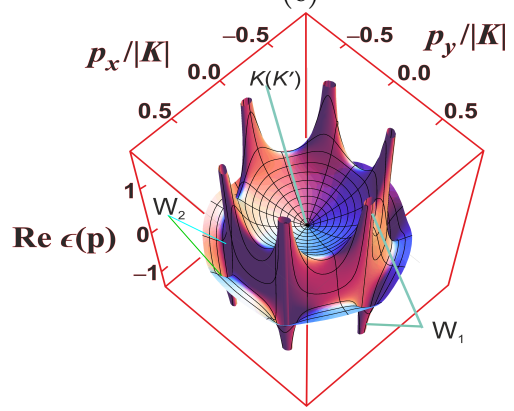

(g)

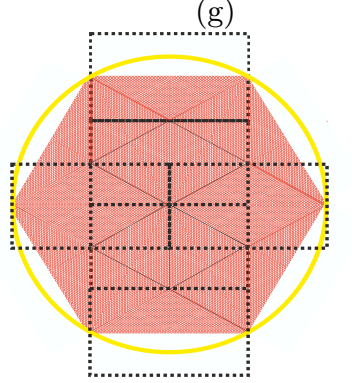

FIG. 2: Scheme of a graphene quantum dot: the $i$-th atom $\mathrm{C}$ is located at the $i$-th site with radius vector $\vec{R}_{i}$, and $\vec{r}$ is a radius vector of the $\mathrm{p}_{z}$-electron relative to the $i$-th lattice site, $O$ is a reference point (a). The graphene quantum-dot supercell used to calculate a quantum dot with torus topology includes $51 \times 51$ primitive cells $(100 \AA \times 150 \AA)$ (b). Splitting of Dirac cone into replicas for graphene in the Dirac-Hartree-Fock quasirelativistic approximation, $q^{4}$ approximation for the exchange interactions. Two $W_{1}, W_{2}$ of the six pairs of Weyl nodes-antinodes: two sources and two sinks and the Dirac valley $K\left(K^{\prime}\right)$ are indicated (c). In (d) and (e) we describe model quantum dots produced by 3 and 6 rhombic unit supercells, respectively. Edges of these quantum dots are zigzag ones. In (f) and (g) we depict model quantum dots produced by 4 and 8 rectangular unit supercells, respectively. The quantum dot in figure (f) holds zigzag- as well as armchair edges. Edges of quantum dot in figure (g) are in armchair configuration. Radius of the yellow color circumference in figures ( $\mathrm{d}-\mathrm{g}$ ) can be interpreted as a radius of quasi-circular quantum dot. Rectangular unit cells are labeled through dotted lines.

Here $N_{d o t}=\left(2 n_{1}+1\right)\left(2 n_{2}+1\right)$ is the total number of graphene hexagonal unit cells within the supercell. For the model quantum dot shown in fig. $2 \mathrm{~b}$ the number of levels $\left(N_{d o t}+1\right)^{2}$ is approximately equal to $6.7 \times 10^{6}$.

In the case of the rhombic unit cell a quantum dot can be generated by, for example, 3 or 6 supercells with one common vertex. Since the vertex is the punctured point of the circle, the topology of these dots is a toroidal one. The quantum dots of this type have zigzag edges only and are presented in figs. $2 \mathrm{~d}, \mathrm{e}$. The torus is obtained by identifying of the opposite sides of the rhombic supercell (at first by gluing with twisting into a cylinder, then by gluing the latter into torus).

The basis vectors of the rhombic supercell are $\vec{C}_{1}=$ $\left(2 n_{1}+1\right) \vec{b}_{1}, \quad \vec{C}_{2}=\left(2 n_{2}+1\right) \vec{b}_{2}$. Therefore quantized wavevectors for the toroidal-type dot are determined as

$$
\begin{gathered}
\left(2 n_{1}+1\right) \vec{b}_{1} \cdot \vec{k}=2 \pi m_{1}, \quad m_{1}=-N_{d o t} / 2+1, \ldots, N_{d o t} / 2 ; \\
\left(2 n_{2}+1\right) \vec{b}_{2} \cdot \vec{k}=2 \pi m_{2}, \quad m_{2}=-N_{d o t} / 2+1, \ldots, N_{d o t} / 2 .
\end{gathered}
$$

The pair of numbers $\left(m_{1}, m_{2}\right)$ entering the formulae (II.3,II.4) is a multi-index of superlattice band. Inversesuperlattice wavevectors $\vec{G}=n_{1} \vec{G}_{1}+n_{2} \vec{G}_{2}$ lie inside the single-layer graphene Brillouin zone. Here $\vec{G}_{1}, \vec{G}_{2}$ are basic vectors of the inverse-superlattice; $n_{1}, n_{2}$ are integers. The superlattice Brillouin zone is very small, and, accordingly, quasiparticle momenta $\vec{p}_{s}, \quad\left(n_{1} \vec{C}_{1}+\right.$ $\left.n_{2} \vec{C}_{2}\right) \cdot \vec{p}_{s}=2 \pi L$, which are due to translational symmetry of the problem, are small also. Here $L$ is integer. Therefore, we can neglect $\vec{p}_{s}$ assuming that the bands are degenerated over $\vec{p}_{s}$ (a dependence on wave number $p_{s}$ is absent). These zero-dimensional (0D) bands is what we name energy levels of our quantum dot model. The first advantage of the proposed "momentum" boundary conditions (II.3, II.4) is in a small number of variants (only two). The nodes of the wave function on the boundary signify that the source of the wave and, correspondingly, an outward current, are absent. The second advantage is that the radius of quantum dot with a given topology determines uniquely a supercell combination forming the dot, and, accordingly, the zigzag and armchair edge configuration of its boundary.

So, we assume that the STM experiment probes the many-electron structure formed under the action of electric field in a region consisting of several supercells. Valence electrons of the structure move in an electric field of core electrons. The latter will be described 
within a pseudopotential method. The pseudopotential corrections are considerably larger than contribution of momenta $\vec{p}_{s}$.

Further, using a folding-zone approach we will calculate pseudopotential-bending 0D-bands of singlelayer graphene in a superlattice potential. In addition, the topology of toroidal or spherical types is a sign of the charge density distribution on circular orbits. The above makes it possible to assign simulated quantum dots to a quasi-circular type. The latter will also be confirmed by the simulation results.

We use a monolayer graphene model $\vec{k} \cdot \vec{p}$-Hamiltonian $\hat{H}_{D}$, in order to determine the energy levels $\epsilon\left(q_{m_{1}, m_{2}}\right)$ of the graphene quantum dot in the folding-zone approximation. Meanwhile, one has to work with the whole Brillouin zone rather than the first one. Let a wavevector $\vec{k}_{m_{1}, m_{2}}$, satisfying either the quantization conditions (II.3) or (II.4), be associated with the reduced wavevector $\vec{q}_{m_{1}, m_{2}}$ as the difference between $\vec{k}_{m_{1}, m_{2}}$ and the nearest Dirac point (valley) $K\left(K^{\prime}\right)$ of Brillouin zone. The Hamiltonian $\hat{H}_{D}$ is obtained within the quasirelativistic Dirac-Hartree-Fock selfconsistent field approach (see [26, 27]). It is a highenergy $\vec{k} \cdot \vec{p}$-Hamiltonian in a $q^{4}$ approximation for the quasirelativistic quantum exchange that the latter is series expanded in the vicinity of the Dirac point $\vec{K}\left(\vec{K}^{\prime}\right)$ on the absolute value of the difference $q=$ $|\vec{p}-\vec{K}|$ of the quasiparticle momentum $\vec{p}$ and $\vec{K}\left(\vec{K}^{\prime}\right)$ up to the terms of $O\left(q^{4}\right)$ inclusively. The deviations of eigenvalues of $\hat{H}_{D}$ from the low-energy massless pseudoDirac fermion model are of the order of $|q|^{4}$. Contrary to non-relativistic graphene model energy levels of the quasirelativistic graphene model are Kramer's doublets. The Kramer's doublet represents itself two levels on which electrons are placed with the opposite signs of spin and in the absence of quantum exchange the levels are degenerated. The relativistic quantum exchange removes degeneration on spin outside the Dirac valley, violating the particle-hole symmetry so that the degeneration of Dirac point is removed outside Dirac valley and six minibands emerge near the Dirac point. Due to the removal of the degeneracy of the Kramer's doublets, each miniband represents a Weyl node-antinode pair. Fig. 2c demonstrates the band structure of the graphene model characterized by the Dirac touching in the point $K\left(K^{\prime}\right)$ and six Weyl node-antinode pairs. Let us note that, as opposed to our quasirelativistic high-energy graphene model, for the low-energy $\vec{k} \cdot \vec{p}$-Hamiltonian of massless pseudo-Dirac fermion a pair of Weyl node and antinode appears after removing of Dirac point degeneration over the pseudospin in three-dimensional topological insulator, so that a Fermi arc along axis $Z$ is observed (see, for example, [28]).

The "folding zone" approximations of quantum-dot wave functions resemble (look similar) to harmonic (sinusoidal) discrete solutions (standing waves) of a quantum mechanical problem on a particle in one- dimensional rectangular well with an infinitely high walls. The wave functions are the bispinors $\psi^{T}=\left(\psi_{\uparrow}, \psi_{\downarrow}\right)=$ $\exp \left\{-i \vec{q}_{m_{1}, m_{2}} \cdot\left(\vec{r}+\vec{R}_{k}\right)\right\}\left(u_{\uparrow}, u_{\downarrow}\right)$ of charge carriers with the energies $\epsilon\left(q_{m_{1}, m_{2}}\right)$. Here " $\uparrow$ " and " $\downarrow$ " designate spinor components with spin "up" and "down" respectively. " $T$ " is the transposition, $u_{\uparrow(\downarrow)}$ are periodic functions: $u_{\uparrow(\downarrow)}\left(\vec{r}+\vec{R}_{k}\right)=u_{\uparrow(\downarrow)}\left(\vec{r}+\vec{R}_{k}+\vec{a}_{S}\right), \vec{a}_{S}$ is a supercell vector.

We sort the energy levels $\epsilon\left(q_{m_{1}, m_{2}}\right)$, in the energy increase order, by introducing an index $i$ for the energies and wavevectors and introduce a formal index $R_{k}$ according to the above presented wave function form. Therefore in what follows, we will denote the energies by $\epsilon_{R_{k}}\left(q_{i}\right)$. We emphasize that the energy does not depend upon $R_{k}$.

Thus, the following set of eigenenergies $\pm \epsilon_{R_{k}}\left(q_{i}\right)$ and eigenstates $\psi_{i}^{(0)}\left(\mp q_{i}, \vec{r}+\vec{R}_{k}\right)=e^{\mp \imath \vec{q}_{i} \cdot\left(\vec{r}+\vec{R}_{k}\right)} u\left(\vec{r}+\vec{R}_{k}\right)$, $u=\left(u_{\uparrow}, u_{\downarrow}\right)^{T}$ for the graphene quantum dot in the folding-zone approximation is

$$
\left\{ \pm \epsilon_{R_{k}}\left(q_{i}\right), \psi_{i}^{(0)}\left(\mp \vec{q}_{i}, \vec{r}+\vec{R}_{k}\right)\right\}_{i, k=1, \ldots, N_{d o t}}
$$

where upper sign "+" is related to electrons and lower sign to "-" corresponds to holes.

In the following section we construct a pseudopotential in which charge carriers of graphene quantum dot move.

\section{B. Continuous graphene quantum dot model with pseudo-potential}

In a graphene monolayer all $\mathrm{p}_{z}$-electrons form pairwise molecular $\pi$-orbitals. $\pi$-electrons located at inside and outside of edges of monolayer patch turn out to be unpaired among $\pi$-electrons, composing an artificial atom of the type of graphene quantum dot. These electrons occupy orbitals of the graphene quantum dot singly and are valence electrons of the artificial atom.

If not all electrons of many-electron system (in our case this is the artificial atom) are coupled, a Hamiltonian of the system includes non-paired-electron potentials that polarize an atomic core (an exchange hole exists). The simplest one-body method of approximate account of the atom polarization in the electric field of the valence electron is based on the Phillips-Kleinman cancellation theorem which states that all electrons, besides on an external orbital, are coupled and the valence electron moves in a pseudo-potential which is added to the atomic core potential [29].

Therefore, one has to add a pseudopotential operator to the high-energy $\vec{k} \cdot \vec{p}$-Hamiltonian $\hat{H}_{D}[30,31]$. Let $|\Psi\rangle$ be a vector of bispinor state in a Hilbert space $\mathcal{H}$ with co-ordinates $\left\langle\vec{X}_{1}, \sigma_{1} ; \ldots ; \vec{X}_{N_{d o t}}, \sigma_{N_{d o t}} \mid \Psi\right\rangle$, written in ordinary space representation through the radiusvectors of charge carriers in the monolayer: 


$$
|\Psi\rangle=\sum_{n_{1}, \ldots, n_{m}=1}^{N_{\text {dot }}} \sum_{\sigma_{n_{1}}, \ldots, \sigma_{n_{m}}} \int d \vec{X}_{n_{1}} \ldots d \vec{X}_{n_{m}}\left|\vec{X}_{n_{1}}, \sigma_{n_{1}} ; \ldots ; \vec{X}_{n_{m}}, \sigma_{n_{m}}\right\rangle\left\langle\vec{X}_{n_{1}}, \sigma_{n_{1}} ; \ldots ; \vec{X}_{n_{m}}, \sigma_{n_{m}} \mid \Psi\right\rangle
$$

Here $\sigma_{i}$ is a spin of $i$-th electron. In this Dirac bracket representation of wave functions for the Fock space [32] the coordinates (wave functions) $\left\langle\vec{X}_{i_{m}}, \sigma_{i_{m}} \mid \Psi\right\rangle \equiv$ $\left(\psi_{m, \uparrow}\left(\vec{X}_{i}\right), \psi_{m, \downarrow}\left(\vec{X}_{i}\right)\right)^{T}=\psi_{m}\left(\vec{X}_{i}\right)$ of $m$-th particle states $\left|\psi_{m}\right\rangle$ in (II.6) are obtained by an action of projectors $\left|\vec{X}_{i}, \sigma_{i}\right\rangle\left\langle\vec{X}_{i}, \sigma_{i}\right|$, satisfying to the resolution of identity: $\hat{I}=\sum_{\sigma_{i}} \int d \vec{X}_{i}\left|\vec{X}_{i}, \sigma_{i}\right\rangle\left\langle\vec{X}_{i}, \sigma_{i}\right|$. Here $\left|\vec{X}_{i}, \sigma_{i}\right\rangle$ and $\left\langle\vec{X}_{i}, \sigma_{i}\right|$ are a vector of the Hilbert space $\mathcal{H}$ and a vector conjugated to it.

The one-body Hamiltonian operator for an electron moving in the quantum dot pseudopotential reads

$$
\hat{H}_{D}\left|\psi_{m}\right\rangle=\left(\epsilon_{m}-\sum_{j=1}^{N_{d o t}} \hat{\epsilon}^{\dagger} P_{j}\right)\left|\psi_{m}\right\rangle
$$

where the second term in the right hand side of eq. (II.7) is the pseudopotential operator $\sum_{j=1}^{N_{\text {dot }}} \hat{\epsilon}^{\dagger} P_{j}$ determined through a hole energy operator $\hat{\epsilon}^{\dagger}$ and a projection operator

$$
P_{j}=\int d \vec{X}_{k} d \vec{X}_{i}\left|x_{k}\right\rangle\left\langle x_{k} \mid \psi_{j}\right\rangle\left\langle\psi_{j} \mid x_{i}\right\rangle\left\langle x_{i}\right|,
$$

coefficient matrixes $\left\langle x_{k}^{\prime}\left|P_{j}\right| x_{i}^{\prime}\right\rangle$ of which have the following form $\left\langle x_{k}^{\prime}\left|P_{j}\right| x_{i}^{\prime}\right\rangle=$ $\left(\begin{array}{cc}\psi_{j \uparrow}\left(\vec{X}_{k}^{\prime}\right) \psi_{j \uparrow}^{*}\left(\vec{X}_{i}^{\prime}\right) & \psi_{j \downarrow}\left(\vec{X}_{k}^{\prime}\right) \psi_{j \uparrow}^{*}\left(\vec{X}_{i}^{\prime}\right) \\ \psi_{j \uparrow}\left(\vec{X}_{k}^{\prime}\right) \psi_{j \uparrow}^{*}\left(\vec{X}_{i}^{\prime}\right) & \psi_{j \downarrow}\left(\vec{X}_{k}^{\prime}\right) \psi_{j \downarrow}^{*}\left(\vec{X}_{i}^{\prime}\right)\end{array}\right) ; \quad \epsilon_{m} \quad$ is $\quad$ a $\quad m-$ th eigenvalue of $\hat{H}_{D}$.

We use a representation where the operator $\hat{\epsilon}^{\dagger}$ is a matrix, which elements $\left\{\epsilon_{k c_{i}}\right\} \equiv\left\{\epsilon_{R_{k}-R_{i}}\left(q_{c_{i}}\right)\right\}$ belong to the set (II.5). Here, on the definition of valence electron, $\epsilon_{k c_{i}}=0$ at $k=c_{i}$. In this representation the equation (II.7) is an equation of motion for the valence $k$-th electron with a radius-vector $\vec{X}_{k}=\vec{r}+\vec{R}_{k}$ :

$$
\begin{array}{r}
\hat{H}_{D} \psi_{k}\left(\vec{r}+\vec{R}_{k}\right)+\sum_{i, c_{i} \neq k}^{N_{d o t}-1} \epsilon_{k c_{i}} P_{c_{i}} \psi_{c_{i}}\left(\vec{r}+\vec{R}_{k}\right) \\
=E_{k} \psi_{k}\left(\vec{r}+\vec{R}_{k}\right), \quad k=1, \ldots, N_{d o t} .
\end{array}
$$

Here $P_{c_{i}}=\int d \vec{X}_{n} \psi_{k}\left(\vec{X}_{n}\right) \psi_{c_{i}}^{*}\left(\vec{X}_{n}\right), \quad \epsilon_{k c_{i}} \psi_{c_{i}}\left(\vec{X}_{k}\right) \equiv$ $\hat{\epsilon}^{\dagger} \psi_{c_{i}}\left(\vec{X}_{k}\right): \epsilon_{k c_{i}} \psi_{c_{i}}\left(\vec{X}_{k}\right)=\int d \vec{X}_{c_{i}}\left\langle x_{k}\left|\epsilon^{\dagger}\right| x_{c_{i}}\right\rangle\left\langle x_{c_{i}} \mid \psi_{c_{i}}\right\rangle$.

Since a quantum dot consists of sufficiently many atoms, one can construct its continuous model in a hydrodynamic limit $\left|\vec{R}_{k+1}-\vec{R}_{k}\right|=a \rightarrow 0, r \rightarrow 0$. A radius-vector $\vec{R}$ of a point in the continuous graphene quantum dot model takes the values of radius-vectors of lattice sites

$$
\vec{R}=\vec{R}_{1}, \vec{R}_{2}, \ldots, \vec{R}_{N_{d o t}}
$$

A derivative $\frac{\partial}{\partial \vec{R}}$ for the continuous graphene quantum dot model is determined in the following way:

$$
\begin{gathered}
\left.\frac{\partial}{\partial \vec{R}} \Psi(\vec{R}) \stackrel{\text { def }}{=}\left\{\frac{\psi_{k}\left(\vec{R}_{k+1}\right)-\psi_{k}\left(\vec{R}_{k}\right)}{\vec{R}_{k+1}-\vec{R}_{k}}\right\}\right|_{k=1} ^{N_{d o t}} \\
=\left.\left\{\frac{\psi_{k}\left(\vec{R}_{k}+\vec{a}\right)-\psi_{k}\left(\vec{R}_{k}\right)}{\vec{a}}\right\}\right|_{k=1, a \rightarrow 0} ^{N_{d o t}},
\end{gathered}
$$

where $\Psi(\vec{R})$ is a graphene-quantum-dot wave function defined by the following expression:

$$
\left.\Psi(\vec{R}) \in\left\{\psi_{k}\left(\vec{r}+\vec{R}_{k}\right)\right\}\right|_{k=1, r=a} ^{N_{d o t}}
$$

Using the definition (II.11) one can determine a convolution $\vec{\sigma} \cdot \frac{\partial}{\partial \vec{R}} \Psi_{v \uparrow(\downarrow)}$ between the derivative of "up" ("down")-spinor component $\Psi_{v \uparrow(\downarrow)}$ and 2D-vector of Pauli matrixes $\vec{\sigma}=\left(\sigma_{x}, \sigma_{y}\right)$ for a $v$-th electron as

$$
\begin{aligned}
& \vec{\sigma} \cdot \frac{\partial}{\partial \vec{R}} \Psi_{v \uparrow(\downarrow)}(\vec{R})=\sum_{i=1}^{2} \sigma_{i} \frac{\partial}{\partial x_{i}} \Psi_{v \uparrow(\downarrow)} \\
& =\left.\sum_{i=1}^{2} \sigma_{i}\left\{\frac{\psi_{v \uparrow(\downarrow)}\left(\vec{R}_{k}+\left(\vec{e}_{i}, \vec{a}\right) \vec{e}_{i}\right)-\psi_{v \uparrow(\downarrow)}\left(\vec{R}_{k}\right)}{\left(\vec{e}_{i}, \vec{a}\right)}\right\}\right|_{k=1, a \rightarrow 0} ^{N_{d o t}} \\
& \equiv \vec{\sigma} \cdot \vec{\nabla}_{\vec{R}} \Psi_{v \uparrow(\downarrow)}(\vec{R}),
\end{aligned}
$$

where $\vec{e}_{i}, i=1,2$ are orthonormal vectors along the coordinate axes $X, Y ;(\cdot, \cdot)$ is a scalar product, $\vec{R}_{i}=x_{i} \vec{e}_{i}$. Then, taking into account that $E_{k}$ is obtained by the action of the operator $i \hbar \frac{\partial}{\partial t}$ on $\psi_{k}\left(\vec{r}+\vec{R}_{k}\right): E_{k} \psi_{k}(\vec{r}+$ $\left.\vec{R}_{k}\right)=i \hbar \frac{\partial}{\partial t} \psi_{k}\left(\vec{r}+\vec{R}_{k}\right)$ the system of equations (II.9) in the hydrodynamic limit can be rewritten as

$$
\begin{array}{r}
\left\{\hat{H}_{D} \psi_{k}\left(\vec{r}+\vec{R}_{k}\right)+\sum_{c \neq k} \epsilon_{k c} P_{c} \psi_{c}\left(\vec{r}+\vec{R}_{k}\right)\right. \\
\left.=E \psi_{k}\left(\vec{r}+\vec{R}_{k}\right)\right\}_{k=1, r \rightarrow a}^{N_{d o t}} .
\end{array}
$$

Using the definitions (II.10 - II.12) and the definition of the projection operator one gets an equation of motion 
for the valence $v$-th electron in a pseudopotential $V_{G Q D}$ :

$$
\hat{H}_{D}\left|\Psi_{v}\right\rangle+\sum_{i=1, c_{i} \neq v}^{N_{d o t}-1}\left|\Psi_{c_{i}}\right\rangle \epsilon_{c_{i}}(\vec{R})\left\langle\Psi_{c_{i}} \mid \Psi_{v}\right\rangle=E\left|\Psi_{v}\right\rangle .
$$

Here $\quad V_{G Q D} \equiv \sum_{i=1, c_{i} \neq v}^{N_{d o t}-1}\left|\Psi_{c_{i}}\right\rangle \epsilon_{c_{i}}(\vec{R})\left\langle\Psi_{c_{i}}\right|, \quad$ a matrix $\epsilon_{c_{i}}(\vec{R})$ is determined by the expression $\epsilon_{c_{i}}(\vec{R}) \in\left\{ \pm \epsilon_{R_{k}-R_{i}}\left(q_{c_{i}}\right)\right\}_{k=1, \ldots, N_{\text {dot }}}$ entering the expression (II.5). A scalar product $\left\langle\Psi_{c_{i}} \mid \Psi_{v}\right\rangle=$ $\sum_{\sigma} \int\left\langle\Psi_{c_{i}} \mid \vec{R}^{\prime}, \sigma\right\rangle\left\langle\vec{R}^{\prime}, \sigma \mid \Psi_{v}\right\rangle d \vec{R}^{\prime}$ of the wave-functions $\left|\Psi_{c_{i}}\right\rangle$ and $\left|\Psi_{v}\right\rangle$ of core and valence electrons of graphene quantum dot and, consequently the operator $V_{G Q D}$ are constructed on a basic set of functions entering the expression (II.5):

$$
\left\langle\Psi_{c_{i}} \mid \Psi_{v}\right\rangle=\sum_{k=1}^{N_{d o t}} \psi^{(0)}{ }_{R_{k}}^{\dagger}\left(\mp \vec{q}_{c_{i}}, \vec{R}_{k}\right) \psi_{R_{k}}^{(0)}\left(\mp \vec{q}_{v}, \vec{R}_{k}\right)
$$

and

$$
\begin{aligned}
& \left\langle\Psi_{c_{n}}\left|V_{G Q D}(\vec{R})\right| \Psi_{c_{m}}\right\rangle \\
& =\sum_{i=1, c_{i} \neq v}^{N_{\text {dot }}-1}\left\langle\Psi_{c_{n}} \mid \Psi_{c_{i}}\right\rangle \epsilon_{c_{i}}(\vec{R})\left\langle\Psi_{c_{i}} \mid \Psi_{c_{m}}\right\rangle \\
& =\sum_{i=1, c_{i} \neq v}^{N_{d o t}-1} \sum_{k, l=1}^{N_{d o t}} \psi^{(0)^{\dagger}}{ }_{R_{k}}\left(\mp \vec{q}_{c_{n}}, \vec{R}_{k}\right) \psi_{R_{k}}^{(0)}\left(\mp \vec{q}_{c_{i}}, \vec{R}_{k}\right) \\
& \times \epsilon_{R_{k}-R_{l}}\left(q_{c_{i}}\right) \psi^{(0)^{\dagger}}{\stackrel{R}{R_{l}}}_{\left(\mp \vec{q}_{c_{i}}, \vec{R}_{l}\right)} \\
& \times \psi_{R_{l}}^{(0)}\left(\mp \vec{q}_{c_{m}}, \vec{R}_{l}\right) .
\end{aligned}
$$

To reveal distinctive features from known potentials, let us find an approximation of the pseudo-potential (II.17) schematically in $\delta$-vicinity of the Dirac point, $\delta \ll 1$ : $q_{c_{n}}, q_{c_{m}}, q_{c_{i}} \rightarrow 0$. To do it, we substitute (II.5) into (II.17), assuming $q_{c_{n}} \rightarrow q_{c_{m}}$ at $r=a$ :

$$
\begin{array}{r}
V_{n, \vec{R}_{s}}(\vec{r})=\lim _{q_{c_{n}} \rightarrow q_{c_{m}}} \pm \sum_{\sigma, \sigma^{\prime}} \sum_{i=1, c_{i} \neq v}^{N_{d o t}-1} \sum_{k, l=1}^{N_{d o t}}|u|_{\sigma}^{2}\left(\vec{r}+\vec{R}_{k}\right) \\
\times|u|_{\sigma^{\prime}}^{2}\left(\vec{r}+\vec{R}_{l}\right) e^{ \pm i\left(\vec{q}_{c_{n}}-\vec{q}_{c_{i}}\right) \cdot\left(\vec{R}_{k}-\vec{R}_{l}\right)} \epsilon_{R_{s}}\left(q_{i}\right) \Theta(r-a), i \neq k .
\end{array}
$$

Here $\vec{R}_{s}=\vec{R}_{k}-\vec{R}_{l}, \Theta(r-a)$ is a Heaviside $\Theta$-function. Changing summation on $i, k, l$, by integration in the expression (II.18) and using the periodicity property of states $u_{\uparrow(\downarrow)}$ we find the pseudopotential in a long-wave approximation:

$$
\begin{array}{r}
V_{n, \vec{R}_{s}}(\vec{r})= \pm \sum_{\sigma, \sigma^{\prime}}|u|_{\sigma}^{2}(r)|u|_{\sigma^{\prime}}^{2}(r) \epsilon_{R_{s}}\left(q_{n}\right) \int e^{ \pm i\left(\vec{q}_{c_{n}}-\vec{q}_{c_{i}}\right) \cdot \vec{R}_{k}} \\
\times \Theta(r-a) d \vec{R}_{k} d \vec{q}_{c_{i}}= \pm(2 \pi)^{2} \sum_{\sigma, \sigma^{\prime}}|u|_{\sigma}^{2}(\vec{r})|u|_{\sigma^{\prime}}^{2}(\vec{r}) \epsilon_{R_{s}}\left(q_{i}\right) \\
\times \Theta(r-a) \Theta\left(q_{c_{n}}-q_{c_{i}}\right), i \neq k .
\end{array}
$$

The expression (II.19) at $q_{c_{n}} \equiv 2 \pi / R \rightarrow 0, \vec{R}=\vec{R}_{s}+\vec{r}$ trends to a pseudopotential in the Dirac point:

$$
\begin{array}{r}
V_{i, R_{s}}(\vec{r})= \pm(2 \pi)^{2} \epsilon_{R_{s}}\left(q_{i}\right) \Theta\left(\lambda_{i}-R\right) \Theta(a-r) \\
\times \sum_{\sigma, \sigma^{\prime}}|u|_{\sigma}^{2}(r)|u|_{\sigma^{\prime}}^{2}(r),
\end{array}
$$

where $\lambda_{i}=\frac{2 \pi}{q_{i}}$. This stair-case-like (cos-shape) potential (II.20) resembles the experimental one shown in fig. $1 \mathrm{~b}$. The Hamiltonian with the approximate pseudopotential of stair-case type (II.20) has a set of quasi-zero energy levels. This feature of the artificial atom is similar to a very high principal quantum number for ultraheavy atoms. The values of radial step $V_{0}$ for all these resonances are close, that explains the experimental data in [7] without invoking speculative assumptions, for example, about the shift of Dirac touchings in the process of STM probing.

Now we solve the following eigenproblem

$$
\begin{aligned}
& \sum_{j}\left\langle\Psi_{c_{n}}\left|\hat{H}_{D}(\vec{R})\right| \Psi_{c_{j}}\right\rangle\left\langle\Psi_{c_{j}} \mid \vec{R}, \sigma\right\rangle \\
& +\sum_{m}\left\langle\Psi_{c_{n}}\left|V_{G Q D}(\vec{R})\right| \Psi_{c_{m}}\right\rangle\left\langle\Psi_{c_{m}} \mid \vec{R}, \sigma\right\rangle=E\left\langle\Psi_{c_{n}} \mid \vec{R}, \sigma\right\rangle .
\end{aligned}
$$

\section{RESULTS AND DISCUSSION}

\section{A. Emergence of quasi zero-energy band}

To fit a derivative $d I / d V$ of STM-current $I$ with respect to bias $V$ it is necessary to calculate LDOS. LDOS reads

$$
\rho(\vec{R}, E)=\sum_{i}\left|\Psi_{i}(\vec{R})\right|^{2} \delta\left(E-\epsilon_{i}\right),
$$

where the summation is performed over all eigenstates of the system. The presence of $\delta$-functions reflects the fact of infinitely small spectral line width of an eigenstate. For a finite spectral line width, we have to assign some decay rate $\gamma_{i}$ to a given eigenstate and use $\delta$-function approximation resulting in Lorentzian spectral line form

$$
\rho(\vec{R}, E)=\sum_{i} \frac{\left|\Psi_{i}(\vec{R})\right|^{2}}{\left|E-\epsilon_{i}+\imath \gamma_{i}\right|^{2}}=\sum_{i} \frac{\left|\Psi_{i}(\vec{R})\right|^{2}}{\left(E-\epsilon_{i}\right)^{2}+\gamma_{i}^{2}} .
$$

In practice, $\gamma_{i}$ are considered as given parameters in a way not to overlap spectrum lines of interest. In our numeric simulation we have chosen $\gamma=10^{-3}$ for upper eigenstates and $\gamma=10^{-5}$ for lower part of eigenstates. The simulation is time consuming for LDOS. It has been performed at a 32 core cluster with MPI parallelization, the simulation time for a single LDOS point is about $10 \mathrm{~min}$. 
(a)

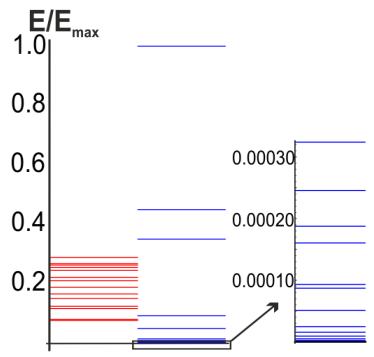

(b)

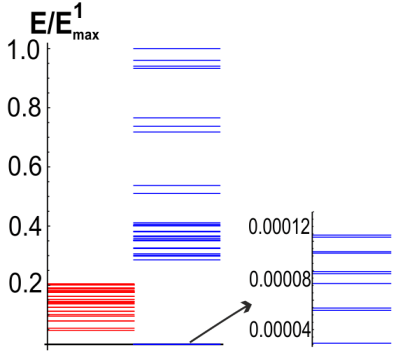

(c)

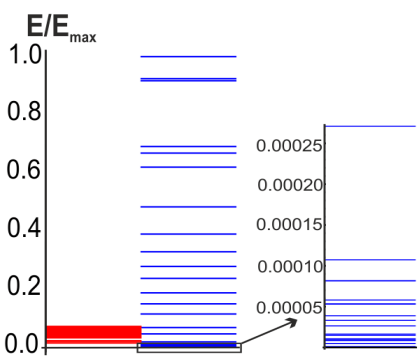

(d)

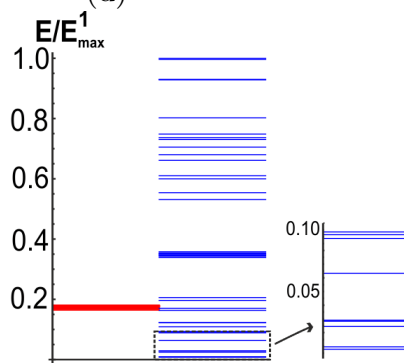

FIG. 3: Energy spectra for graphene quantum dots with topology $S^{1} \times S^{1}$ (a, b) and $S^{2}$ (c, d) on low- (a, c) and high-energy scales (b, d). In each figure the left and right spectra correspond to the calculations in the folding-zone and pseudopotentialapproximation approximations respectively. All spectra are normalized to the largest value of the right spectrum.

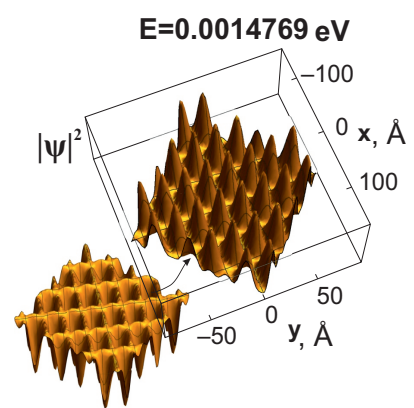

$E=0.000762951 \mathrm{eV}$
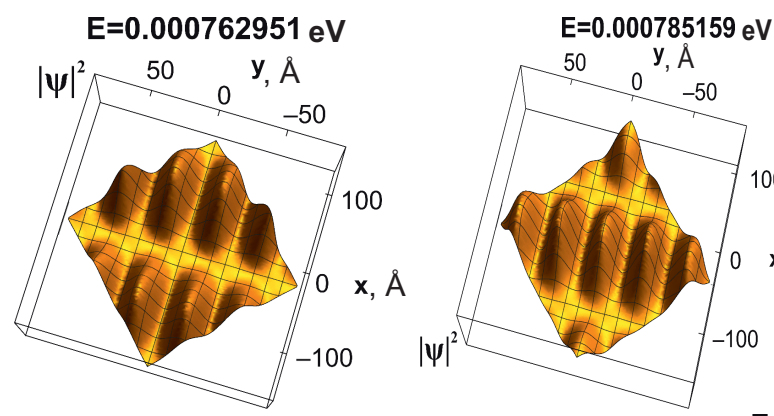

$50 \mathrm{y}, \AA$

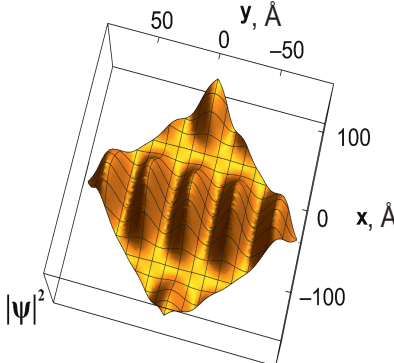

$E=0.00149691 \mathrm{eV}$

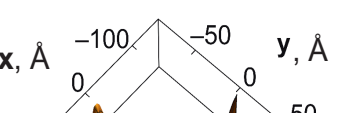

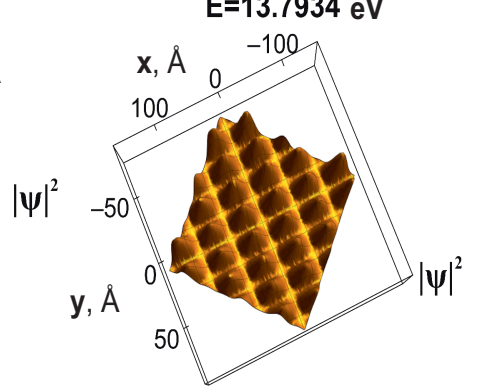

$E=0.00115898 \mathrm{eV}$
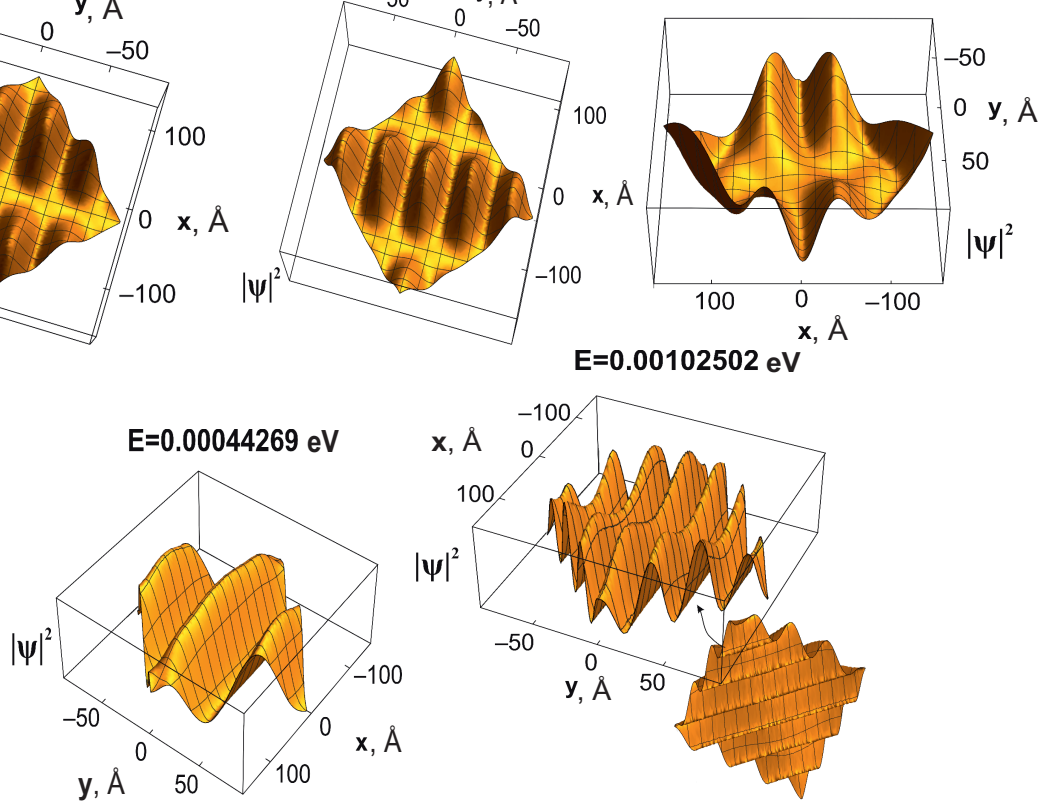

FIG. 4: The pseudopotential confinement of $S^{1} \times S^{1}$-dot states. Squares $|\Psi|^{2}$ of the absolute values of wave functions are presented. The states are localized on resonant (upper row) and non-resonant (middle row) trajectories of torus or are delocalized due to Klein transitions (bottom row) for different energies $E$. For the levels $E=0.0014769,0.00102502 \mathrm{eV}$ we represent both sightseeing "from up" and "from down". 
LDOS determined by the formula (III.2) depends on three variables: two space variables and the energy. At least, in modern STM experiments all directions of charge-current vector for the dot contribute into the tunnel current. Therefore, it is necessary to perform contour-averaging procedure. We perform averaging over some contour in $\vec{r}$-space accounting of points equivalent from the viewpoint of lattice symmetry. For the toroidal graphene quantum dot we choose for averaging contours $C_{T}$ around a center of $2 \mathrm{D}$-toric-like region from three rhombuses closed sequentially on each other accounting of graphene lattice symmetry. For the spherical graphene quantum dot, we choose for averaging circles $C_{S}$ with increasing radii around a point in the center of rhombus side accounting of graphene lattice symmetry. The contours $C_{T}$ and $C_{S}$ are topologically equivalent to closed contours on torus and sphere.

We study LDOS on two energy scales. For the torus and sphere topologies, we choose the low- and highenergy ranges $0.24 \div 0.92 \mathrm{eV}$ and $0.68 \div 2.94 \mathrm{eV}$ and, respectively, $0.24 \div 1.22 \mathrm{eV}$ and $2.98 \div 3.26 \mathrm{eV}$. The scales were obtained in the following way. For the lowenergy range, we get the solutions of the conditions of quantization (II.3), (II.4) for $m_{1}, m_{2} \in[-25,25]$, which corresponds to the choice of 40 lowest energy levels from 2601. For the high-energy interval, we determine the solutions of the conditions of quantization (II.3), (II.4) for $m_{1}, m_{2} \in[-12,12]$, which corresponds to the choice of 40 lowest energy levels from 625 . Comparing the structures of the levels $E_{D}\left(\vec{k}_{i}\right)$ and $E^{(0)}\left(\vec{k}_{i}\right), i=1, \ldots, 40$ for the pseudo-Dirac graphene quantum dot model and our graphene quantum dot model with Weyl nodes-antinodes, respectively, in the folding-zone approximation we conclude that the Weyl nodes-antinodes decrease the degree $p_{d}$ of level degeneration. The effects of topology and symmetry manifest themselves in different maximum values of $p_{d}$ for the sphere and torus topologies in the folding-zone approximation. The maximum values $\max \left(p_{d}\right)$ are equal to 4 and 12 for the pseudo-Dirac spherical and toroidal quantum dot models, respectively. In the case of Weyl nodes-antinode pairs $\max \left(p_{d}\right)$ takes values of 4 and 6 for the spherical and toroidal quantum dot models, respectively. The pseudopotential completely removes the degeneration of levels $E^{(0)}\left(\vec{k}_{i}\right), i=1, \ldots, 40$ and the resulting spectrum $E_{i}^{(1)}, i=1, \ldots, 40$ consists of two bands. The lower energy band formed by levels located near the zero energy $E=0$ is a quasi-zeroenergy band, as one can see in fig. 3. The spectra for both toroidal and spherical quantum dots in the pseudopotential approximation possess a very narrow quasi-zero-energy band.

\section{B. Toroidal quantum dot}

Fig. 4 demonstrates squares $\left|\Psi_{i}\right|^{2}=\sum_{j=1}^{2} \Psi_{i j}^{*} \Psi_{i j}$ of the absolute values of $i$-th spinor wave functions. "Hills" in fig. 4 represent electron states. A bottom view of $3 \mathrm{D}$ plot is shown in inset, upper row, fig. 4. "Wells" in the inset represent hole states. At definite energies corresponding to resonant trajectories on torus, the orbitals are similar to those for Bohr atoms when the length of the closed orbit is an integer number of wavelengths. Due to the zero-curvature of torus the motion of charge carrier on such orbits is stable. There also exist other energy levels (fig. 4, bottom row). On them the phases of wave functions are multiples of $\pi \pm 2 \pi n, n=0,1, \ldots$. The latter feature makes their similar to transmission resonances of the Klein tunneling process. Charge carriers delocalized due to the Klein tunneling can be considered in analogy with atomic states as valence ones. The localized states are similar to the core electrons of atom.

According to fig. 5, the main feature of the structure of energy levels for the toroidal quantum dot is the presence of self-similar energy bands located subsequently one after another on the energy scale. The atom-like structure (LDOS) for some bands marked by dashed lines in fig. 5 is realized only for toroidal graphene quantum dots, from the geometric viewpoint, due to the absence of the curvature of a torus. These levels are occupied by electrons (holes) with wave functions of the type of Bohr atom orbital (fig. 4 (upper row)). The electron (hole) density can be localized also in the case of non-resonant torus trajectories due to a constructive interference (see fig. 4(middle row)).

For the toroidal graphene quantum dot, the chargecarrier density is absent on the quasi-zero-energy band due to a destructive interference of the states. The latter fact leads to the formation of a pseudogap between the hole and electron bands, providing the absence of the electron density at the center of the quantum dot, like in the case of an ordinary atom.

\section{Spherical-type quantum dot}

The probability density of states for the quantum dot with sphere topology is shown in fig. 6. According to a form of the wave functions, the charge carriers are localized due to a constructive interference in the case of non-resonant sphere configurations (fig. 6a) or are the transmission resonances delocalized in a limited region at the state phases multiple to $\pi$ (fig. $6 \mathrm{~b}-\mathrm{e}$ ). The resonances of the $S^{2}$-dot reside in the quasi-circle like region located either inside one rhombic cell, or in two conjugate rhombuses. Therefore, the sphericaltype dot can be classified as a quasi-circular quantum dot, electrostatically confined in one or two supercells bordering on each other. The action of centrifugal forces stipulated by the sphere curvature destroys Bohr atom 

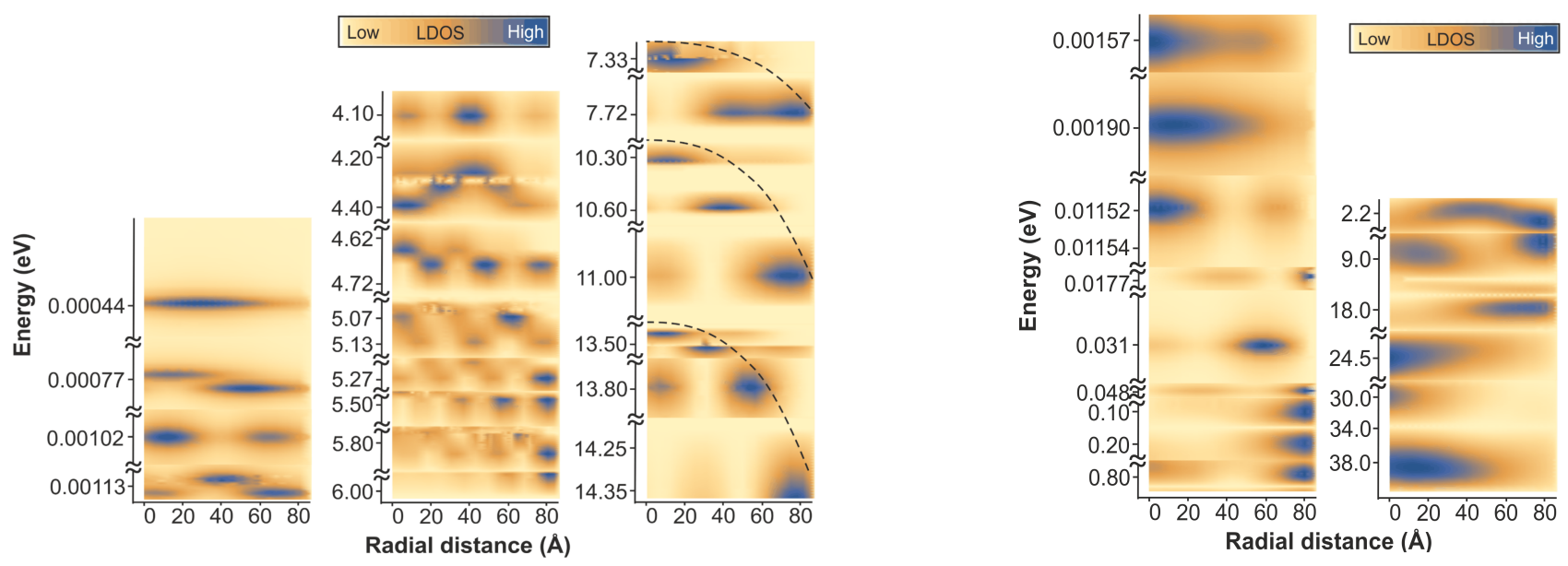

FIG. 5: LDOS of a toroidal quantum dot in bending bands. (to the left) LDOS for the states possessing high energies in the folding-zone approximation, (to the right) LDOS for the states possessing low energies in the folding-zone approximation. The self-similar bands are marked by dashed lines.

orbitals, formed on resonant trajectories (with an integer number of wave periods on the trajectory). However, such features of atomic structure as localized "core" charge carriers and delocalized valence states remain. The "transmission resonances" play a role of the valence states.

Fig. 7 demonstrates that the structure of energy levels for the spherical-type graphene quantum dot hosts self-similar energy bands placed subsequently one after another on the energy scale and the levels can hold a multi-resonance structure. This feature resembles that of the toroidal quantum dot. The electron (hole) density with such a structure is confined by staircase-like (cosshape) potential as one can see in figs. 7b,c. Opposite to the case of toroidal quantum dot, the charge-carrier density in spherical quantum dot resides also on the quasi zero-energy band (see fig. 7a). The localized non-resonant electron (hole) configurations shown in middle row, fig. 4 and fig. 6a are observed for both spherical and toroidal graphene quantum dots.

\section{Comparison with experimental data}

We now compare the measured experimentally and theoretically predicted distributions of the charge density in electrically confined graphene quantum dots.

In [7], graphene p-n junctions with an atom-like structure have been epitaxially grown up to a size of $8 \mathrm{~nm}$ along radius $R$ on a copper support. Figs. 8a, c demonstrate different theoretical predictions for LDOS of the small-radius p-n junction whose spectroscopic map of the radially averaged derivative $d I / d V$ of a STMcurrent $I$ with respect to the bias $V$ is shown in fig. 8b. Their features are brought together in Table 1. The value of the first level has been chosen as an origin for the energy. We take the graphene dielectric constant $\epsilon=2.0$. Our estimation of the graphene dielectric constant is consistent with the experimental one equal to 2.2 for graphene carrier concentration $n \sim 10^{9} \mathrm{~cm}^{-2}$ [33]. We observe the following differences between the spectroscopic map, LDOS for our toroidal graphene junction model, and LDOS estimated on the assumption that the confining $\mathrm{p}-\mathrm{n}$-junction potential is a radial step one. Our levels prediction is extremely precise. Their structure and arrangement are also in perfect agreement with the experimental ones. The second level of our model is unscreened. Higher laying states are screened. The fitting based on the low-energy pseudoDirac model Hamiltonian with a radial step potential gives not only values which are very different from the experimental ones, but also an incorrect resonance structure because the 3rd level of the junction is doubled. As one can see, a serious drawback of the theoretical approach [7] is also the absolute inability to describe correctly the form of resonances: the theoretically predicted resonances are localized in energy (very narrow) and are spread in space. But the energy levels of experimental quantum dot are strongly widened in energy and localized in space. The states of toroidal graphene junction under the action of the pseudopotential are strongly spatially localized similarly to atomic electron shells and are broadened in energy $E$ (see fig. 8) as in the experiment. It is easy to see that the experimental confining potential is a parabolic type only. Our numerical results indicate also that a parabolic potential approximates the confining potential.

We conclude that the strength of the electric field created by electrons of the lowest 1st level is not sufficient for the production of electron-hole pairs. But, the electrical field strength of the electrons of the lowest two 1st and 2nd levels is strong enough to produce the electron-hole pairs. Therefore, a screened confining pseudopotential acts on electrons of the $3 \mathrm{rd}$ and subsequent levels. 
(a)

(b)
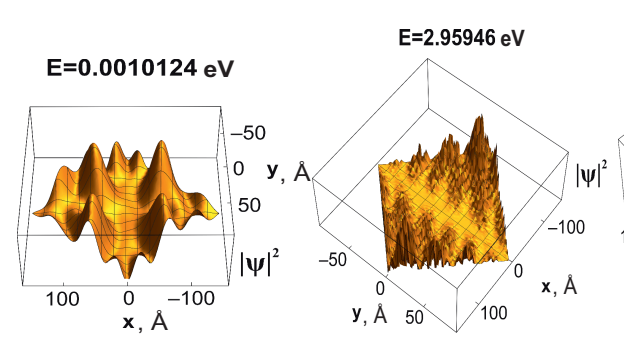

(c)

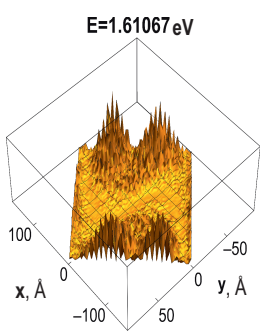

(d)

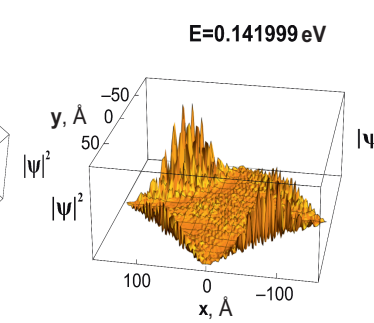

(e)

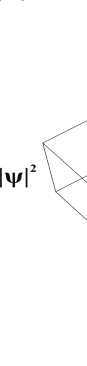

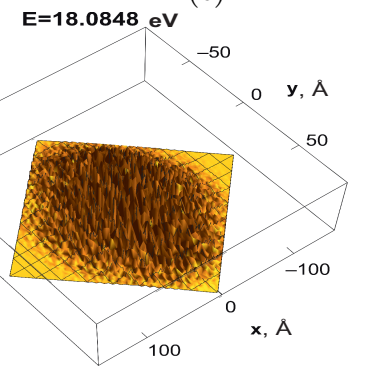

FIG. 6: The pseudopotential confinement of $S^{2}$-dot states with different energies $E$. Squares $|\Psi|^{2}$ of the absolute values of wave functions are presented. The states are localized on non-resonant trajectories of sphere (a) or are delocalized due to Klein transitions (b-e).

(a)

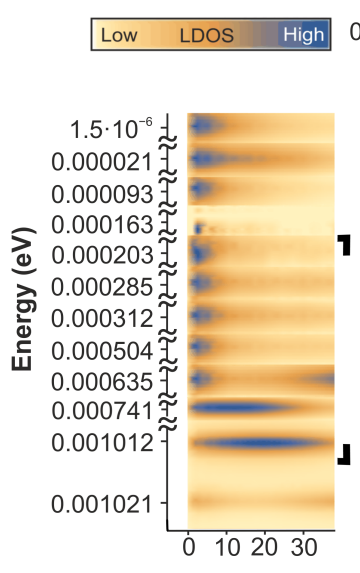

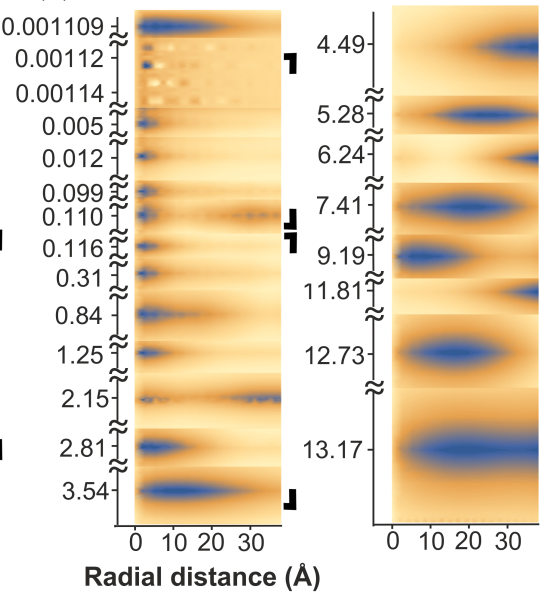

(b)

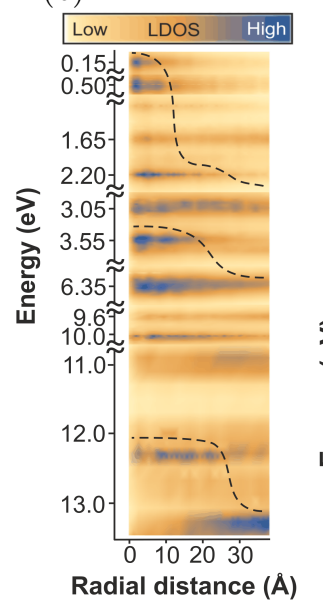

(c)

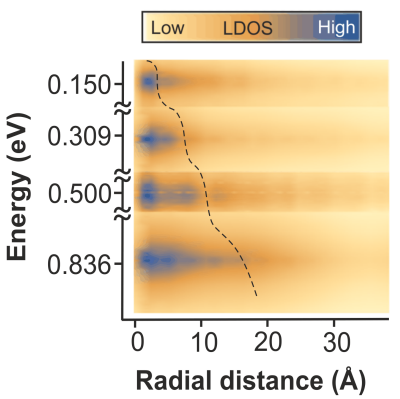

FIG. 7: LDOS of a spherical quantum dot in bending bands. (a) LDOS for the states possessing low energies in the foldingzone approximation; (b) LDOS for the states possessing high energies in the folding-zone approximation; (c) a band in the overlapping energy range. Self-similar bands are marked by dashed lines and bold angles.

In [6], large graphene quantum dot with a radius $150 \mathrm{~nm}$ were fabricated by the local embedding of a gate in a graphene/h-BN heterostructure on $\mathrm{SiO}_{2}$. Let us compare a theoretical LDOS for the continuous model of quantum dot with torus topology (fig. 9a) and a second derivative $\frac{d^{2} I}{d V^{2}}$ of a STM-current $I$ measured as a function of the bias $V$ and the radial distance from the center of the circular graphene $\mathrm{p}-\mathrm{n}$ junction deposited on $\mathrm{BN} / \mathrm{SiO}_{2}$ (fig. 9b). Our numerical calculation of LDOS predicts the levels with a multiresonance structure. These levels are completely analogous to multiresonance structures of the corresponding experimental levels. An attempt has been made to explain these experimental data using a model of massless pseudo-Dirac fermions in parabolic potential in [6]. It turns out that some theoretical levels predicted by this low-energy pseudo-Dirac theory of large graphene p-n junction are experimentally unobservable. Besides, some levels experimentally observable are absent in the theory. For example, the pseudo-Dirac model of a circular graphene quantum dot does not predict the highest experimental energy level of about $80-85 \mathrm{mV}$ (this level is absent). Moreover, all levels with the same numbers of resonances predicted by this pseudo-Dirac fermion model Hamiltonian are arranged in pairs. However, the doubling is not confirmed experimentally (see figs. 9a,b). The main drawback of the pseudo-Dirac model is as follows: at a bias from 0 and lower, the number of levels in the experimental is twice less than that predicted theoretically.

Finally, let us consider the low-energy region from -50 to $-100 \mathrm{mV}$ (corresponding theoretically calculated levels are placed at $-50 \mathrm{meV}$ and lower). Contrary to LDOS in the pseudo-Dirac model, the experimental LDOS and the theoretical LDOS confined by the pseudopotential are redistributed to the border of graphene quantum dot. Respectively, their hole density is polarized.

Let us compare the experimental data on tipinduced electrically confined graphene quantum dots in a graphene monolayer covering a $30-\mathrm{nm}$-thick BN flake on 
Table I: Assignment of theoretically predicted quantum-dot resonances to experimental data. The resonances are arranged in ascending order of distance from the center of the quantum dot and are measured from the deepest level.

\begin{tabular}{|l|l|l|l|l|}
\hline \hline \multirow{2}{*}{$\begin{array}{l}\text { Level } \\
\text { number }\end{array}$} & \multirow{2}{*}{$\begin{array}{l}\text { A number of } \\
\text { resonances }\end{array}$} & \multicolumn{3}{|c|}{ Resonance energies, eV } \\
\cline { 3 - 5 } & Experiment & Our theory & Theory in $[7,34]$ \\
\hline \hline 1 st & one & 0 & 0 & 0 \\
\hline $2 \mathrm{nd}$ & one & 0.108 & $0.109^{*}$ & 0.064 \\
\hline $3 \mathrm{rd}$ & two & 0.213 & 0.194 & $0.13,0.175$ \\
\hline $4 \mathrm{th}$ & one & 0.46 & 0.51 & 0.29 \\
\hline $5 \mathrm{th}$ & 0.42 & 0.48 & 0.415 \\
\hline \hline
\end{tabular}

(a)

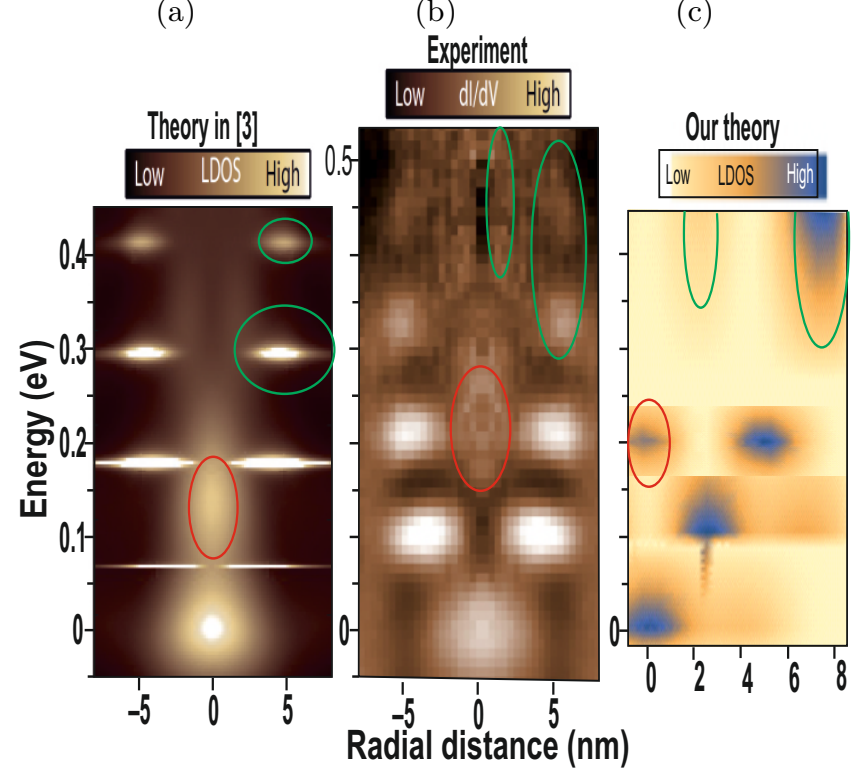

FIG. 8: Spectrum of atom-like small graphene quantum dot. The background correction (chemical potential) $\mu$ has been subtracted from the raw data leading to a shift in the energy so that the energy of lowest resonance in the potential well is zero one. (a) - calculated results presented in [7] using the radial step potential. (b) - the dependence of derivative $d I / d V$ of a STM-current $I$ with respect to the bias $V$ as a spectroscopic map of graphene quantum dot deposited on a copper support [7]; (c) - our results calculated with the graphene dielectric constant $\epsilon=2$ except of two lowest levels with $\epsilon=1$, the unscreened band of the continuous toroidal model graphene quantum dot under the action of a pseudopotential is placed in the range $13.35 \div 14.35 \mathrm{eV}$.

graphite with our numerical calculations of quantum dot with sphere topology. The sphere has a nonzero curvature leading to a staircase-like (cos-shape) pseudopotential in fig. $7 \mathrm{c}$. This result is in complete agreement with the experimental fitting shown in fig. 1b. The pushing out of electrons by the "centrifugal force" onto the quasi-zeroenergy band is revealed as the nonzero electron density on its levels.

By compensating the action of a pseudopotential, (a)

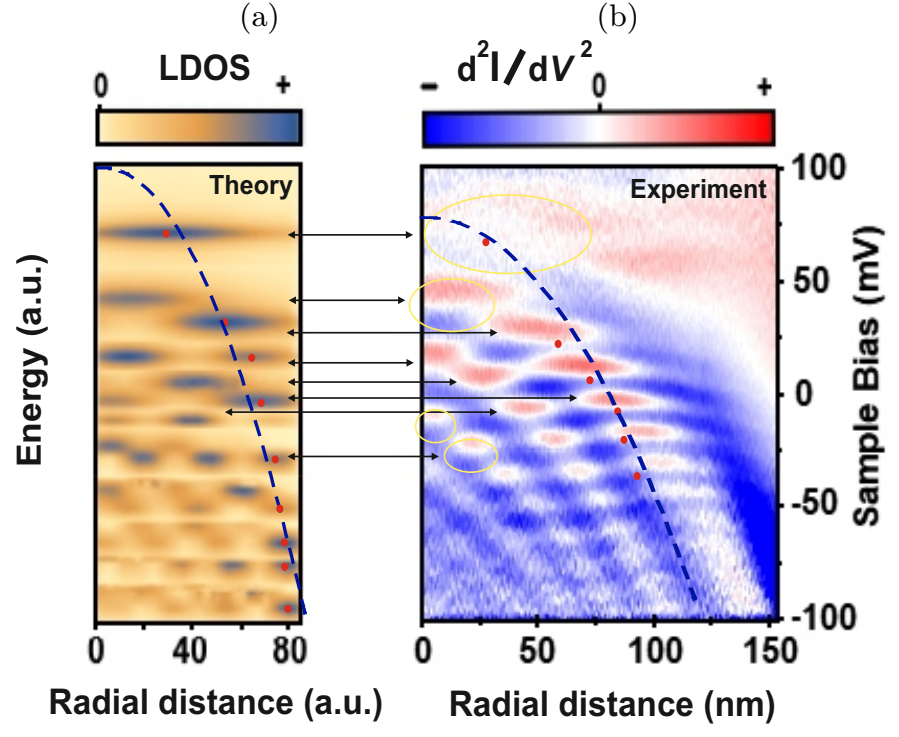

FIG. 9: Energy levels of spatially distributed states of a large graphene quantum dot. (a) Theoretically simulated LDOS in the bands of states confined by a pseudopotential for the continuous model of quantum dot with torus topology. (b) Second derivative $\frac{d^{2} I}{d V^{2}}$ of a STM-current $I$ measured as a function of the bias $V$ and the radial distance from the center of a circular graphene $\mathrm{p}-\mathrm{n}$ junction deposited on $\mathrm{BN} / \mathrm{SiO}_{2}$. Lines with arrows indicate the theoretical bands, coinciding with experimental ones. Some resonances are encircled by yellow oval to improve perception. A fitting parabolic potential is indicated by a dashed line.

for example, by the Lorentz force, one can achieve a weak bending of the bands by a pseudopotential with the grouping of nondegenerate levels as in the folding-zone approximation. According to our theory of graphene $p-n(n-p)$ junctions with sphere topology, the multiplicity of energy-level degeneration in the foldingzone approximation is $p_{d}=2,4$, like in the experiment [4]. Moreover, the multiresonance distribution of LDOS over the energy levels for the graphene quantum dot with sphere topology in fig. 7 leads to the experimentally observed independence of charging peak sequence on the distance between the cantilever STM tip and the 
quantum-dot center. The above discussion allows us to classify this electrostatically confined quantum dot on the $\mathrm{BN} /$ graphite support as a spherical one.

Thus, the theoretical predictions of the continuous model of quantum dot in monolayer graphene with one Dirac point and 6 pairs of Weyl nodes-antinodes under the action of a pseudopotential not only explain, but show the excellent quantitative agreement with various experiments.

\section{CONCLUSION}

In this work, we have utilized the rhombic and rectangular graphene supercells to describe the confinement of electrons/holes. Discrete and continuous atom-like models of graphene electrically confined quantum dot with the topology of torus and sphere have been proposed. The charge carriers are confined through polarization effects also. The electron/hole states localized and delocalized in the pseudopotential are calculated in a continuous approximation. The polarization of graphene quantum dot due to the pseudopotential "pushes out" the energy levels, by removing their degeneration.

The resonances of toroidal graphene quantum dot are partitioned into three types of resonances: strong and weak localized "core electrons" and wave packages for delocalized "valence electrons". We assume that the strong localization may be a consequence of commensurate frequencies of the Hamiltonian system on the torus. The strong localized states of the toroidal quantum dot are formed as in a Bohr atom. For the toroidal quantum dot, the electron (hole) density is absent on the quasi-zero-energy band due to the zero curvature of torus. This leads to the formation of a pseudogap between the hole and electron bands, which ensures the absence of the charge density at the center of the quantum dot, like at the center of Bohr atom. Accordingly, the toroidal-type quantum dot is an artificial atom with Bohr orbits.

Unlike the toroidal quantum dot, the spherical graphene quantum dot hosts multiresonances, whose charge density is present at its quasi-zero-energy band. Accordingly, three spherical dots of the hexagonal quantum dot play the role of the nucleus of the artificial atom.

To summarize, the quasirelativistic model graphene $\mathrm{n}-\mathrm{p}$ (p-n) junctions with the supercell pseudopotential which electrically confines electrons (holes) have been proposed. This potential can bend energy levels in the parabolic way. For graphene quantum dots with the sphere topology, the parabolic potential is additionally modulated in stair-case (cos-shape) wise. This approach explains the main features of a local distribution of charge carrier density for the quantum dots observed in various STM-experiments. The advantage of the approach we develop is the ability not only to correctly predict the arrangement of levels, but also to describe the phenomenon of polarization in graphene quantum dots.
[1] Jia Zhang, Shu-Hong Yu, Materials Today. 19, 382 (2016)

[2] N.N. Bogolubov, A.V. Soldatov, S.P. Kruchinin, Quantum Matter. 4, 352 (2015)

[3] V. V. Kudryashov, Int. J. Nonlin. Phen. in Compl. Sys. 12, 199 (2009)

[4] N.M. Freitag, L.A. Chizhova, P.Nemes-Incze, C.R. Woods, R.V. Gorbachev, Y. Cao, A.K. Geim, K.S. Novoselov, J. Burgdörfer, F. Libisch, M. Morgenstern, Nano Lett. 16, 5798 (2016)

[5] Y. Jiang, J. Mao, D. Moldovan, M.R. Masir, Gu. Li, K. Watanabe, T. Taniguchi, Fr.M. Peeters, E.Y. Andrei. Nat. Nanotechnology. 12, 1045 (2017); J. Mao, Y. Jiang, D. Moldovan, Gu. Li, K. Watanabe, T. Taniguchi, M.R. Masir, Fr.M. Peeters, E.Y. Andrei. Nature Phys. 12, 545 (2016).

[6] J. Lee, D. Wong, J. Velasco Jr, J.F. Rodriguez-Nieva, S. Kahn, H.-Z. Tsai, T. Taniguchi, K. Watanabe, A. Zettl, F. Wang, L.S. Levitov, M.F. Crommie, Nature Physics. 12, 1032 (2016)

[7] Ch. Gutiérrez, L. Brown, Cheol-Joo Kim, Jiwoong Park, A.N. Pasupathy, Nature Physics. 12, 1069 (2016)

[8] Yu. Zhao, J. Wyrick, F.D. Natterer, J.R. Nieva, C. Lewandowski, K. Watanabe, T. Taniguchi, L. Levitov, N.B. Zhitenev, J.A. Stroscio, Science. 348, 672 (2015)

[9] G. Giavaras, F. Nori, Phys. Rev. B 85, 165446 (2012)

[10] P. A. Maksym, M. Roy, M. F. Craciun, S. Russo, M. Yamamoto, S. Tarucha, H. Aoki, J. Phys. Conf. Ser. 245,
$012030(2010)$

[11] A. Hansen, F. Ravndal. Physica Scripta. 23, 1033 (1981)

[12] A.H. Castro Neto, F. Guinea, N.M.R. Peres, K.S. Novoselov, A.K.Geim. The electronic properties of graphene. Rev. Mod. Phys. 81, 109 (2009)

[13] A. Calogeracos, N. Dombey, K. Imagawa. His. At. Nucl. 159, $1275(1996)$

[14] Pr. Hewageegana, V. Apalkov, Phys. Rev. B. 77, 245426 (2008)

[15] A. Matulis, Fr.M. Peeters. Phys.Rev. B. 77, 115423 (2008).

[16] T D Linh Dinh et al. Quasibound states in single-layer graphene quantum rings, 2018 J. Phys.: Condens. Matter 30315501

[17] K. Nakada, M. Fujita, G. Dresselhaus, and M. S. Dresselhaus. Phys.Rev. B54, 17954 (1996); H. P. Heiskanen, M. Manninen, and J. Akola,New J. Phys.10,103015 (2008). A.V. Rozhkov, G. Giavaras, Y.P. Bliokh, V. Freilikher, F. Nori. Physics Reports. 503, 77 (2011).

[18] M. Grujic, M.Zarenia, A. Chaves, M.Tadic, G.A. Farias, F.M. Peeters. Phys. Rev. B 84, 205441 (2011)

[19] Z.Z. Zhang, K. Chang, F.M. Peeters, Phys.Rev. B. 77, $235411(2008)$

[20] P. Potasz, A.D. Güçlü, P. Hawrylak. Phys. Rev. B. 81, 033403 (2010).

[21] K.A. Ritter, J.W. Lyding. Nat. Mater. 8, 235 (2009). 
[22] G. Pal, W. Apel, L. Schweitzer. Phys. Rev. B. 84, 075446 (2011).

[23] J.-R. Wang and G.-Z. Liu, J. Phys.: Condens. Matter 23, $155602(2011)$

[24] L.L. Li, M. Zarenia, W. Xu, H.M. Dong, F.M. Peeters. Exciton states in a circular graphene quantum dot: Magnetic field induced intravalley to intervalley transition. Phys. Rev. B 95, 045409 (2017)

[25] S. Reich, C. Thomsen, J. Maultzsch. Carbon nanotubes. Basic Concepts and Physical Properties. (WILEY-VCH Verlag GmbH \& Co. KGaA, Wenheim, 2004)

[26] H.V. Grushevskaya, G. Krylov, J. Mod. Phys. 5984 (2014).

[27] H. V. Grushevskaya and G. Krylov, Int. J. Nonlin. Phen. in Compl. Sys. 18, 266 (2015)

[28] H.-Zh. Lu, Sh.-Q. Shen. Front. Phys. 12(3), 127201
(2017)

[29] J.C. Phillips, L. Kleinman, Phys. Rev. 116, 287 (1959)

[30] V.A. Fock, Fundamentals of Quantum Mechanics (Mir Publishing, 1978)

[31] H. Krylova, L. Hurski, Spin polarization in strongly correlated systems. (LAP Lambert Academic Publishing, 2013)

[32] A. Altland, B. Simons. Condensed Matter Field Theory. (Cambridge University Press, New York, 2010)

[33] D.C. Elias, R.V. Gorbachev, A.S. Mayorov, S.V. Morozov, A.A. Zhukov, P. Blake, L.A. Ponomarenko, I.V. Grigorieva, K.S. Novoselov, F. Guinea, A.K. Geim, Nat.Phys. 8, 172 (2012)

[34] H.Ch. Nguyen, N.T.T. Nguyen, V.L. Nguyen, J. Phys. C.M. 29, 405301 (2017) 